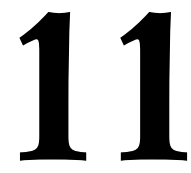

\title{
EL ESTUDIANTE INMIGRANTE Y SU INCLUSIÓN EN LA UNIVERSIDAD ESPAÑOLA
}

\author{
(INMIGRANT STUDENT AND THEIR INCLUSION IN THE SPANISH \\ UNIVERSITY)
}

$\mathrm{M}^{\mathrm{a}}$ Paz Lebrero Baena y $\mathrm{M}^{\mathrm{a}}$ del Pilar Quicios García UNED

\section{RESUMEN}

Este artículo es producto de un proyecto de investigación financiado por el MICINN que intenta visualizar las dificultades que los inmigrantes universitarios manifiestan tener para obtener éxito en sus estudios. Se trata de una investigación no experimental de carácter descriptivo y correlacional que utiliza como unidad de análisis el inmigrante universitario matriculado en una carrera en distintas universidades públicas españolas. Emplea metodologías cuantitativas y cualitativas, recogiéndose la información a través de un cuestionario con respuestas de escala tipo Lickert, convenientemente validado, y una serie de grupos de discusión para obtener datos de carácter cualitativo.

Además de hacer un breve recorrido por la literatura al respecto y de exponer los datos numéricos y cualitativos del Estudio, se van a ofrecer algunas medidas inclusivas para intentar solventar cada uno de los problemas académicos que ha manifestado tener esta población. Estos apoyos inclusivos indicados podrían, igualmente, ser útiles para cubrir las necesidades que puedan presentar, en los próximos años, los estudiantes europeos que se movilicen por las universidades españolas.

\section{ABSTRACT}

This article is the product of a research project financed by the Spanish Ministry of Science and Innovation in an attempt to visualise the difficulties 
immigrant university students claim to experience in succeeding at university in Spain. This non-experimental, descriptive, correlational research uses as its unit of analysis the immigrant university student pursuing a degree, in different Spanish public universities. The study employs quantitative and qualitative methodologies, gathering information through a questionnaire with answers on a properly validated Lickert-type scale and a series of discussion groups to get qualitative data.

In addition to brief review by the literature in the matter, the numerical and qualitative data of the Study, are exposed and present some inclusive measures offered to endeavour to solve each of the academic problems the target population claimed to have met. These supportive measures could likewise be useful in covering the needs that European students may display in upcoming years as they move more freely through Spanish universities.

\section{INTRODUCCIÓN}

El presente estudio es el resultado parcial de un proyecto de investigación financiado por el MICINN a través del Programa de Estudios y Análisis 2008 referenciado EA-2008/0030 (Pérez y otros, 2008).

El tema de la inmigración universitaria ha resultado ardua y complicada, debido, por una parte, a la cantidad de variables que inciden en ella, como son la gran variabilidad numérica - debido a la movilidad geográfica- y su consiguiente inestabilidad cuantitativa, el cambio de actividad laboral constante - casi permanente- que complementa su devenir diario y el estar cursando estudios entre los inmigrantes académicos.

A todo esto es preciso añadir las distintas facetas de los estudiantes universitarios, como son el haber diseñado su proyecto migratorio sobre la búsqueda de una mejora laboral y haber colocado como objetivo de su viaje al exterior el adquirir un mayor bagaje académico y cultural.

Antes de abordar el estudio empírico, haremos una breve referencia a los antecedentes sobre el tema.

\section{BREVE RESUMEN DE LA LITERATURA SOBRE EL TEMA}

En el año 2004 sonó el pistoletazo de salida de una nueva formación universitaria, la diseñada siguiendo las directrices marcadas por el EEES. A fin de prepararse para ello, la Unión Europea no ceja de dar pasos en esta dirección. Uno de ellos ha sido el declarar el año 2008 Año europeo del diálogo intercultural bajo el lema "Unidos en la diversidad". Así, para conocer el pun- 
to del que parte la realidad social europea sobre diálogo intercultural, este año, Europa ha elaborado el "Eurobarómetro" en la Discriminación en la Unión Europea 2008 (Comisión Europea, 2008).

España, como país integrante de la Unión Europea, ha tomado parte en el estudio realizado y así, desde el 18 de febrero al 22 de marzo de 2008 , ha respondido a 1.004 entrevistas de las 26.746 efectuadas en toda Europa.

Parece oportuno acotar más el estudio teórico al territorio español, por ser el marco geográfico en el que se va a tratar de poner en marcha un tipo de universidad diferente, de orientación inclusiva. Para ello hay que conocer la sensibilización, compromiso y actitudes que tiene la sociedad española hacia la población inmigrante. Entre los problemas que le preocupan se encuentra la inmigración -en un lugar intermedio-, según los estudios realizados por el Observatorio Español del Racismo y la Xenofobia (Ministerio de Trabajo e Inmigración, 2008a) entre los meses de mayo y julio de 2008.

Por su parte, el Instituto de Estudios Sociales Avanzados de Andalucía -IESA - dependiente del CSIC, por encargo de la Dirección General de Integración de los Inmigrantes, llevó a cabo un estudio para conocer la sensibilización real existente hacia la población inmigrante en el país (Pérez y Desrues, 2007). Destacamos los siguientes porcentajes: El 69\% considera la diversidad cultural más bien positiva. El 61,8\% considera excesivo el número de inmigrantes asentados en España. El 90,2\% rechazaría trabajar con inmigrantes. El 41,7 \% se relaciona a diario con la población inmigrante.

La pregunta clave en este campo para nuestra investigación era: ¿Considera aceptable que un alumno o alumna pueda ser excluido de la universidad o centro educativo por ser inmigrante? Las respuestas obtenidas son bien elocuentes y permiten pensar en poner en marcha una universidad de tipo inclusivo con garantías de éxito. El 62,2\% lo considera inaceptable; el $28,5 \%$ aceptable y el $8,3 \%$ no sabe o no contesta.

Fernández y Terrén (2008:15) señalan que en la inmigración no está la solución a los problemas sociales y económicos. Por su parte, Arrimadas y Hernando $(2007,31)$ hacen ver la riqueza social que este fenómeno mundial produce en la sociedad del país de acogida.

Desde otro punto de vista Guillén y Ontiveros (2007) realizaron un estudio valorando la riqueza mutua que produce la inmigración —-poblaciones venidas de lejos - para el país diana y para la población que migra, así como la contribución para "crear un acervo valiosísimo de capital humano".

Finalmente, destacar que, a lo largo de los años 2003 a 2006 se desarrolló el Proyecto "INCLUES" o Llaves para una educación inclusiva y cogni- 
tiva con el objetivo de crear una red de profesionales interesados en desarrollar esta modalidad educativa. Participaron entidades de países tales como: Bélgica, Reino Unido, Italia, Noruega, Letonia, Rumania, República Checa, y España.

Sirvan estas referencias para reflejar la importancia de la temática, si bien, en este breve trabajo no podemos abordar más en profundidad cada uno de los temas implícitos en él, aisladamente, tales como la inclusión educativa, la inmigración en España y los datos —abandono/permanenciade los estudiantes universitarios.

Consideramos que hemos de centrarnos en la confluencia de dichos aspectos, respondiendo al título propuesto en el trabajo: inclusión-inmigrantesuniversitarios.

\subsection{Universidad inclusiva e inmigración}

Cada vez más suenan las voces a favor de la universidad inclusiva. En la Clausura de la Conferencia Regional para la Educación Superior en América Latina y el Caribe - CREES - celebrada en Argentina (Gil, 2008) se concluyó diciendo que la Educación Superior debe comprometerse a superar y contrarrestar las deficiencias estructurales y las Universidades tienen que asumir un rol protagónico y estratégico en el que el fomento de políticas de inclusión y de desarrollo sea un hecho activo y eficaz.

La globalización también ha llegado al mundo de las políticas educativas. España no es una excepción. Si bien hay opiniones críticas - Oliver y otros (2004) —, la realidad nos muestra que el crecimiento de estudiantes universitarios sigue aumentando lentamente en las universidades españolas. Consideran que con la integración de los inmigrantes en las aulas universitarias españolas, los estudiantes se acostumbrarán a vivir en una natural diversidad cultural y religiosa propia de un contexto multiétnico o, cuando menos, multicultural. Proponen un reto social de futuro interesante, afirmando que si se consiguiese que los inmigrantes de segunda generación se integrasen en la clase media española, sus hijos tendrían más posibilidades de acceder a nuestra Universidad, sin necesidad de que ésta fuera plenamente inclusiva.

Muestran la Universidad como el cenit de la integración social, laboral, económica, cultural, afectiva y real de la política migratoria. En esta línea afirman que para aumentar la matrícula universitaria entre los estudiantes inmigrantes resulta fundamental que estos estudiantes estén motivados y apoyados por sus padres mientras cursan los niveles anteriores en España. 


\subsection{Datos de los estudiantes de origen inmigrante en la universidad}

Dentro del avance de las Estadísticas Universitarias del alumnado que proporciona el MEC (2008a:19-20) encontramos los siguientes datos:

\begin{tabular}{|l|r|r|r|r|r|}
\hline & \multirow{2}{*}{ Total } & \multicolumn{2}{|c|}{ Extranjeros } & \multirow{2}{*}{ UE (27) } & $\begin{array}{c}\text { Otros } \\
\text { extranjeros }\end{array}$ \\
\cline { 3 - 4 } & & \multicolumn{1}{|c|}{ Total } & \multicolumn{1}{c|}{$\%$} & & \\
\hline Estudiantes de $1^{\text {er }}$ y $2^{\circ}$ ciclo & 1.389 .384 & 32.138 & $2,3 \%$ & 10.710 & 21.428 \\
\hline Estudiantes de Máster & 33.021 & 7.496 & $22,7 \%$ & 1.567 & 5.929 \\
\hline Estudiantes de Doctorado & 77.654 & 16.996 & 21,9 & 3.637 & 13.359 \\
\hline
\end{tabular}

Cuadro 1. Estudiantes universitarios extranjeros

Es necesario tener en cuenta los países de origen de estos estudiantes.

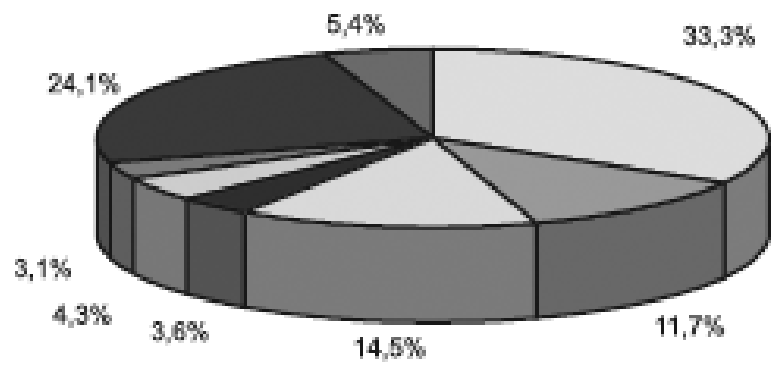

\begin{tabular}{|c|c|c|c|}
\hline $\begin{array}{l}\text { 口UE(27) } \\
\text { america del Norie }\end{array}$ & $\begin{array}{l}\text { 口Resito Europa } \\
\square \text { América Central }\end{array}$ & $\begin{array}{l}\text { 口Nerie Africa } \\
\text { América del sur }\end{array}$ & $\begin{array}{l}\text { DRasilo Africa } \\
\text { 口Asia y Ocean/a }\end{array}$ \\
\hline
\end{tabular}

Gráfico 1. Origen de los estudiantes inmigrantes universitarios,

Centrándonos, exclusivamente, en la población de nuestra investigación, es decir, inmigrantes en edad formativa superior (de 18 a 34 años), agrupados por sexo, origen y nivel de estudios podemos afirmar que, dependiendo de los países de origen y del sexo del estudiante universitario, se puede disponer - en el mejor de los casos-de un porcentaje cercano al $40 \%$ de población inmigrante en situación de formarse universitariamente en España. Este porcentaje de población inmigrante, potencialmente estudiante universitario, es un importante activo que es preciso atender, si se pretende desarrollar una universidad de tipo inclusivo. 
Si consideramos los estudiantes extranjeros que terminan sus estudios, Graduados en el Sistema Universitario Español y los correspondientes a la Unión Europea (UE-27), encontramos los siguientes datos (MEC, 2009,23):

\begin{tabular}{|c|c|c|c|c|c|c|c|c|}
\hline & \multicolumn{4}{|c|}{ Matriculados } & \multicolumn{4}{|c|}{ Graduados } \\
\hline & \multirow{2}{*}{ Total } & \multicolumn{3}{|c|}{ Extranjeros } & \multirow{2}{*}{ Total } & \multicolumn{3}{|c|}{ Extranjeros } \\
\hline & & Total & $\%$ total & UE-27 & & Total & $\%$ total & UE-27 \\
\hline Total estudiantes & 1.504 .276 & 65.581 & $4,4 \%$ & 19.283 & 225.109 & 10.764 & $4,8 \%$ & 2.816 \\
\hline Estudiantes de $1^{\mathrm{er}}$ y $2^{\circ}$ ciclo & 1.354 .875 & 36.562 & $2,7 \%$ & 12.917 & 187.813 & 2.598 & $1,4 \%$ & 2.009 \\
\hline Estudiantes de Grados & 18.353 & 668 & $3,6 \%$ & 198 & - & - & - & - \\
\hline Estudiantes de Másteres Ofic. & 49.799 & 11.392 & $22,9 \%$ & 2,383 & 13.796 & 2.868 & $21,0 \%$ & 674 \\
\hline Estudiantes de Doctorado & 77.249 & 16.959 & $22,0 \%$ & 3.791 & 23.499 & 3.268 & $22,4 \%$ & 1.133 \\
\hline
\end{tabular}

Cuadro 2. Estudiantes extranjeros matriculados y graduados

Puede concluirse que la universidad, entre su alumnado de origen inmigrante, acoge a estudiantes de los diversos niveles universitarios y les proporciona su correspondiente Título de reconocimiento.

La UNED (2009), tanto por su carácter de universidad nacional extendida a través de sus Centros Asociados por todo el territorio nacional y parte del internacional, como por la metodología que despliega, resulta una de las opciones más accesibles y fáciles para que el estudiante de origen inmigrante pueda acceder, con garantías de éxito, a una titulación universitaria.

\subsection{Referencias legislativas del tema}

Por una parte, centrándose exclusivamente en el análisis de la ley que regula el funcionamiento de las universidades la Ley Orgánica de Universidades (Jefatura del Estado, 2001) se observa que, en su exposición de motivos, aparte de la declaración de intenciones, no se recoge expresiones como educación inclusiva, estudiantes extranjeros, alumnos extranjeros, inmigrantes, estudiantes inmigrantes. El vacío encontrado en la Ley de Universidad fue uno de los motivos que propició el nacimiento de este Proyecto de Investigación en el mundo universitario.

En el ámbito europeo, la Comisión Europea (2009) a través de la Dirección General de Justicia, Libertad y Seguridad (DE) (EN) (FR), lleva a 
cabo la realización de este Espacio con el objetivo de garantizar la libre circulación de personas y ofrecer un elevado nivel de protección a los ciudadanos. La concreción reposa en los Programas de Tampere (1999-2004) y La Haya (2004-2009). Corresponde a la vez al régimen comunitario y al régimen intergubernamental desde el Tratado de Amsterdam.

Por otra parte, son interesantes los derechos fundamentales en la Unión Europea que guardan relación con el Marco Conceptual de este Proyecto de Investigación, así como las aportaciones legislativas que se desprenden del mismo: desde el Convenio Europeo del Estatuto de Trabajadores Inmigrantes (24/11/1977) hasta las conclusiones del Consejo Europeo de Tesalónica (19-22/06/2003). Sin embargo, la limitación del trabajo no nos permite desarrollar.

\subsection{Necesidades de los estudiantes universitarios}

En línea con nuestro Estudio, Fernández (2006) dirigió un Proyecto de Investigación sobre Necesidades de los estudiantes universitarios ante la realidad del Espacio Europeo de Educación Superior: Una propuesta de detección y apoyo personalizado; clarificador para extrapolar de manera sistemática las necesidades que pueden tener los estudiantes inmigrantes matriculados en las Universidades Españolas.

Con él se pretendió aportar, claramente, una serie de apoyos dirigidos a todos aquellos estudiantes universitarios que más los necesitasen. Algunas de las conclusiones del estudio, importantes para este trabajo son las siguientes:

- La presencia de alumnos inmigrantes en las universidades es una realidad minoritaria.

- Para atender a este alumnado es necesario ofrecer servicios y estrategias docentes que faciliten su aprendizaje.

- Las universidades deben aumentar los apoyos a los inmigrantes a través de convenios con otras organizaciones o instituciones.

La plena vigencia de estas conclusiones han tenido una duración cercana a dieciocho meses, puesto que en enero de 2008 la UNED, dentro del Marco de Colaboración con el Ministerio de Trabajo y Asuntos Sociales -MTAS_- sacó a la luz una serie de trabajos compilados como Estudio sobre las demandas del colectivo de estudiantes inmigrantes matriculados en la UNED dirigido por Camarero (2007). 


\section{ESTUDIO EMPÍRICO}

En líneas generales, esta investigación se orienta a promover la inclusión social y laboral del colectivo de inmigrantes universitarios. Concretamente, se pretende responder a la pregunta/problema siguiente. ¿La universidad española favorece al estudiante inmigrante una propuesta inclusiva?

\subsection{Objetivos del estudio}

Se trata de conseguir los siguientes objetivos:

- Identificar el perfil de la población inmigrante universitaria, es decir, conocer sus características personales, demográficas y socioculturales.

- Examinar los motivos que a las poblaciones inmigrantes asentadas en España a realizar este tipo de enseñanzas.

- Establecer los factores que inciden en el éxito de los estudios: intereses, expectativas, antecedentes académicos, recursos utilizados.

- Establecer las posibles causas que llevan a los inmigrantes a abandonar la formación universitaria.

- Identificar los recursos de apoyo que precisan y demandan para obtener éxito.

- Diseñar recursos de apoyo que faciliten buenas prácticas, orientadas a reducir el abandono, conseguir el éxito académico y propiciar la inclusión social.

\subsection{Hipótesis del estudio y variables}

Para dar respuesta a estos objetivos nos planteamos la siguiente hipótesis: Cuanto más inclusiva sea la Universidad, más éxito tendrá el estudiante inmigrante en ella matriculado y mayor ascenso cultural tendrá la sociedad en la que se desarrolle su capital humano.

La investigación que ha posibilitado este Estudio ha tenido un carácter descriptivo y correlacional. Su unidad de análisis ha sido el estudiante inmigrante matriculado en una carrera en una universidad pública española. 
Las variables independientes tenidas en cuenta han sido la dimensión contextual, personal, social y académica. La variable dependiente se ha concretado en el éxito académico (superación de al menos el 50\% de las asignaturas) o el fracaso (abandono al comienzo de los estudios o durante el proceso) de los estudiantes atendiendo a las calificaciones obtenidas y el número de titulados.

\subsection{Población y muestra}

El universo de la investigación lo ha compuesto todos los inmigrantes matriculados en estudios universitarios de primer y segundo ciclo en el curso 2008-2009 en universidades públicas españolas (29.636). Este universo se repartía entre universidades presenciales (26.754) y no presenciales (2.882). La muestra de estudio se formó con estudiantes de la Universidad Autónoma de Madrid, la Universidad Autónoma de Barcelona, la Universidad Pablo de Olavide de Sevilla y la UNED. Las muestras de estudio de cada universidad se obtuvo aplicando la fórmula para poblaciones finitas (Bugeda, 1974).

Con estas poblaciones se realizó un muestreo estratificado y las unidades de análisis se seleccionaron aleatoriamente, con un margen de error \pm 2,5\% (Tagliacarne, 1991). Para reducir los posibles errores se seleccionó una muestra invitada representativa de los colectivos analizados. Se enviaron 1.890 cuestionarios mediante correo electrónico y se recibieron 453 respuestas, valores que representan un $24,62 \%$.

\subsection{Recogida de datos: Instrumentos utilizado y proceso de aplicación}

Dadas las ventajas que ofrece el instrumento del cuestionario, se recurre a él como instrumento principal. Para elaborar la primera redacción se siguieron los pasos habituales, tales como definir objetivos, mapa conceptual de variables y selección de preguntas. Definitivamente se presentaron 53 preguntas cerradas con una escala Lickert de 4 valores para sus respuestas. Por otra parte, se decidió presentar preguntas cerradas en su mayoría, con algunas cuestiones abiertas. Los campos que formaron el cuestionario fueron: características personales y contextuales de la muestra, motivos por los que se emigró a España, vivencias y dificultades encontradas en la universidad, recursos personales, materiales y ambientales en la universidad, medidas de apoyo docente necesitadas por el estudiante, causas de abandono y expectativas de futuro. 
En marzo de 2009 se realizó una aplicación previa del cuestionario piloto a 42 estudiantes universitarios de la UNED y 36 de la Universidad Pablo de Olavide de Sevilla. Igualmente se comprobó la fiabilidad y validez del instrumento a través del coeficiente de correlación de alfa de Cronbach y el paquete estadístico SPSS $(17,0)$ para realizar los cálculos numéricos obteniéndose un valor de 0,857 . La validez del cuestionario se comprobó mediante la validez de contenido sobre los 47 items y, además, se contó con la opinión de 14 expertos de las distintas universidades implicadas.

La aplicación definitiva del cuestionario se realizó entre abril y mayo de 2009, recogiéndose los datos presencialmente, por correo postal y electrónico. Estas respuestas se analizaron bajo el enfoque cuantitativo y cualitativo.

En el enfoque cuantitativo se realizó el análisis de tipo inferencial mediante contrates con ANOVA, con la prueba " $\mathrm{t}$ " de student y con la prueba "ji cuadrado". Igualmente se aplicó el análisis multivariado con el análisis factorial y el análisis discriminante. En el análisis factorial, mediante la extracción de componentes principales, se ha optado por la rotación VARIMAX, con el fin de matizar las correlaciones de todas las variables con cada uno de los siete factores principales seleccionados en el análisis y que agrupan la información principal del cuestionario: "Causas de emigración" “Apoyos personales al estudio" "Apoyos de material al estudio" "Comprensión y expresión oral y escrito" "Capacidad y expectativas para finalizar los estudios superiores" "Causas del abandono" y "Edad".

En el enfoque cualitativo se utilizaron cuadros, matrices, diagramas, planos de análisis intentando unir objetividad en contenidos de cariz intersubjetivo.

Finalmente, se interpretaron las respuestas combinando datos cuantitativos y cualitativos para exponer los resultados.

\section{PRESENTACIÓN DE LOS RESULTADOS}

A continuación se van a enumerar los resultados obtenidos de los cuestionarios de tipo cuantitativo y de los grupos de discusión realizados, con el fin de ampliar los fríos datos estadísticos con aportaciones de naturaleza cualitativa. Se ha optado por una interpretación descriptiva e interpretativa para facilitar una información detallada de los diferentes aspectos que se consideran de interés. 
a) Aspectos personales. El sujeto que ha aportado las siguientes informaciones ha sido el estudiante inmigrante universitario-tipo. Joven de entre 20 y 30 años $(40,6 \%)$, fundamentalmente de sexo femenino $(71,3 \%)$ que se inclina, mayoritariamente, por estudios de larga duración: licenciaturas $(53,1 \%)$. Poblaciones universitarias ya en sus países de origen $(44,2 \%)$ que representan un alto ascenso cultural respecto al nivel académico alcanzado por sus padres (el 17,2\% de las madres de los estudiantes encuestados habían realizado estudios universitarios así como el $23,4 \%$ de sus padres).

b) Causas de la emigración y dificultades encontradas en España. En una escala de valoración de 1 a 4, las causas más valoradas a la hora de decidir abandonar su país de origen, fue el lenguaje $(2,40)$, poder realizar estudios universitarios $(2,30)$ y buscar un porvenir mejor a través de los estudios $(2,22)$.

c) Universidad. La mayor dificultad encontrada en la Universidad española, valorada en un grado medio/alto, ha sido la compatibilidad de los estudios con el trabajo.

d) Causas de abandono. Los datos extraídos del estudio cuantitativo fijan entre las causas de abandono de los estudios- ordenadas porcentualmente- causas laborales, económicas, nivel académico exigido, motivos familiares y problemas administrativos. El estudio cualitativo realizado con esta misma población indica como motivos por los que principalmente se han abandonado los estudios las siguientes problemáticas: la organización y el desarrollo del currículum, la gestión del tiempo de estudio personal, las carencias académicas que se tienen, cuestiones personales, problemas sociales, económicos, afectivos e incompatibilidad estudio-trabajo.

e) Deficiencias. En el desarrollo de la investigación se ha profundizado en las microdeficiencias que han llevado a dejar la formación universitaria; así, entre los aspectos resumidos con la expresión "problemas organizativos y desarrollo del currículum" se encierra la falta de motivación en la que han caído sin ser reactivada por el influjo del profesorado, problemas para conseguir los contenidos del programa de estudios, escasez o brevedad de sesiones personales de tutoría tanto presenciales como telemáticas.

f) Factores de éxito. No todos los estudiantes universitarios de origen inmigrantes se ven abocados a abandonar su carrera formativa. Un gran grupo de ellos tiene éxito en sus estudios. Ese éxito lo van consiguiendo desplegando, a diario, las siguientes habilidades metodológicas: conocer modelos de exámenes anteriores $(85,75 \%)$, contar con apoyos telemáticos (85\%), desarrollar toda su capacidad intelectual $(82,75 \%)$, orientarse por compañeros de cursos superiores $(81,75 \%)$ y encontrarse afectivamente estable sabiéndose aceptado por sus compañeros $(81,25 \%)$. 
g) Necesidad de apoyos. Los estudiantes que tomaron parte en la investigación, con independencia de los resultados académicos alcanzados, señalaron la conveniencia de contar con apoyos educativos útiles para cada estudiante (es decir, educación personalizada en la institución universitaria), apoyo para expresar sus ideas por escrito, apoyo para expresar sus ideas oralmente, apoyo para comprender el lenguaje del profesor, en definitiva técnicas instrumentales básicas de aprendizaje.

h) Recursos didácticos y tecnológicos. El 96.5\% de los encuestados tiene ordenador en casa y su nivel de formación en informática es medio/alto. Por otra parte, los apoyos educativos que reciben son valorados muy positivamente, con un grado muy alto, mientras que valoran en grado bajo/muy bajo la necesidad de apoyos educativos para la mejora de las calificaciones.

i) Ayudas recibidas para el estudio. Los alumnos financian mayoritariamente sus estudios universitarios con su trabajo - valorado en un grado alto/ muy alto-, mientras que la financiación con dinero familiar se ha valorado en un grado bajo/ muy bajo.

j) Expectativas sobre los estudios universitarios. La mayoría de los inmigrantes tienen expectativas altas-muy altas de conseguir un trabajo adecuado a su formación, así como expectativas altas de finalizar la carrera.

Conocidas tanto las causas que llevan a los estudiantes inmigrantes universitarios a abandonar sus estudios como las ayudas que esas mismas poblaciones requieren para tener éxito en los estudios superiores, resulta fácil diseñar mecanismos educativos que suplan las deficiencias de los primeros apoyándose en la experiencia comprobada por el grupo exitoso.

En la investigación desarrollada se siguió indagando este aspecto y volvió a dársele la voz a las poblaciones involucradas que señalaron necesitar una serie de apoyos educativos.

\section{PROPUESTA DE APOYOS INCLUSIVOS PARA UNIVERSITARIOS INMIGRANTES}

Centrándose en el campo educativo, está la necesidad que este país tiene de reducir el índice de abandono de estudios universitarios y la reducción del coste económico de cada puesto académico.

Los jóvenes inmigrantes universitarios solicitan la puesta en marcha de una Universidad de tipo inclusivo, la Comunidad Científica sabe de su éxito, la sociedad necesita jóvenes inmigrantes formados universitariamente, las 
empresas los buscan, la Constitución Española apuesta por la igualdad de oportunidades, la Declaración Universal de Derechos Humanos la avala. La Universidad Latinoamericana también está interesada en este modelo formativo. Así, la clausura de la Conferencia Regional para la Educación Superior en América Latina y el Caribe - CREES - celebrada en Argentina (Gil, 2008) concluyó afirmando que la Educación Superior debe comprometerse a superar y contrarrestar las deficiencias estructurales y las Universidades tienen que asumir un rol protagónico y estratégico en el que el fomento de políticas de inclusión y de desarrollo sea un hecho activo y eficaz.

El éxito académico se genera, en una primera instancia, por el conocimiento real de las capacidades intelectuales, técnicas y volitivas de cada uno de los estudiantes. Estas capacidades, deberían ser conocidas y asumidas antes de matricularse en la formación universitaria. Los estudiantes, tanto inmigrantes como autóctonos, provenientes de la Enseñanza Secundaria Obligatoria conocen sus capacidades reales gracias a la labor realizada por los Departamentos de Orientación de los Centros de Secundaria. De acuerdo con las mismas, estos estudiantes deberían haberse matriculado en titulaciones abordables sin mayores problemas.

En el caso de que el estudiante no conozca realmente sus competencias, precisaría, antes de iniciar su carrera, efectuar pruebas tanto de competencia curricular como de actitudes y aptitudes intelectuales para conocer la realidad de la que parte. Estas pruebas deberían realizarse en los COIEs de las Universidades, conocedores de esta problemática. Los COIEs de las Universidades son entes prácticamente desconocidos por los estudiantes en los primeros días en la Universidad.

Actualmente desde ellos se están diseñando planes de acogida con el objeto de orientar a esta población en su proceso de matriculación, impartiéndoles cursos de formación telemática e información puntual. Si desde estos Centros de Orientación se consiguiesen realizar las correspondientes evaluaciones psicológicas, instructivas, intelectivas y de rendimiento, previas al acceso a la Universidad, se abarataría mucho el coste de cada plaza universitaria, se reduciría el abandono estudiantil, así como la psicosis, neurosis y depresión estudiantil de naturaleza exógena.

Ubicado cada estudiante en la titulación más conveniente para sus capacidades y disposiciones, otro de los factores que asegura el éxito en los estudios es el dominio de las técnicas instrumentales precisas. En los planes de estudio de todas las titulaciones universitarias han de incluirse una serie de asignaturas que permitan conocer al estudiante las técnicas instrumentales básicas que se le van a requerir en la carrera y el nivel de exigencia que se le va a solicitar. Esas asignaturas se deberían desarrollar en el primer mes 
del curso de la titulación. Se ejercitarían de manera intensiva a lo largo de todo ese mes, con un contenido secuenciado y estratificado en dificultad. Deberían ser de carácter obligatorio su ejecución y contabilizar en el expediente del estudiante como asignaturas de libre configuración. El despliegue de este apoyo sería eficaz para todos los estamentos de la docencia universitaria y serviría, igualmente para marcar el nivel de exigencia de la institución.

Con el estudiante formado instrumentalmente e informado del nivel de exigencia que le va a solicitar la institución en la que pretende formarse universitariamente, se va concretando la realidad a la que tendrá que enfrentarse el universitario a lo largo de su periodo estudiantil. Estas medidas, siendo imprescindibles para abordar con éxito cualquier tipo de estudio, se dan por obvias y no se diseñan en casi ningún plan de estudios.

Otro apoyo específico para los estudiantes inmigrantes son los cursos de expresión y comprensión lectora, escrita y oral. En muchas universidades europeas se solicita a los estudiantes extranjeros un nivel determinado de la lengua de instrucción. En las universidades españolas, medir esta competencia lingüística, entre los estudiantes inmigrantes, es un hecho complejo puesto que, la mayoría de los matriculados en ella son de origen hispanoamericano, con lengua materna común.

Este punto de partida confunde a los estudiantes, pues, consideran que van a ser instruidos en un lenguaje que dominan. La realidad es otra bien distinta, una gran parte de los estudiantes latinos manejan el castellano en su nivel coloquial pero presentan grandes carencias y lagunas en el castellano académico más riguroso, exhaustivo y unívoco. La solución a este problema pasaría por introducir, entre las asignaturas que se cursaran en el primer mes del curso, la asignatura de libre configuración pero obligada realización: expresión oral, escrita y lectora. Esta asignatura podría cursarse tanto en castellano como en la lengua propia de la Comunidad Autónoma a la que pertenezca la Universidad en la que se haya matriculado el estudiante.

Como solución global para estas poblaciones inmigrantes debería diseñarse un curso cero de introducción a la Universidad, curso de nivelación o curso de técnicas instrumentales universitarias. En ese curso, de un semestre de duración, se incidiría en la lectura comprensiva, expresión escrita, correcta ortografía, sintaxis y semántica castellana o de la lengua autonómica que fuera a necesitar y en la adquisición de un variado vocabulario académico. Igualmente se introduciría al estudiante en la alfabetización digital, en el manejo de bibliotecas y archivos así como de fuentes documentales diversas. Se trabajarían técnicas de estudio y de trabajo cooperativo, dinámicas de grupos, habilidades sociales e inteligencia emocional. 
En definitiva, el objetivo de este semestre sería dotar al estudiante universitario de origen inmigrante de todas aquellas herramientas que le permitieran adquirir y expresar, correctamente, todos los conocimientos, destrezas, habilidades y valores que la instrucción universitaria despliega hacia todos sus estudiantes, con independencia de su lugar de nacimiento.

El estudiante foráneo, en una universidad inclusiva, debe sentirse miembro de la sociedad estudiantil de la que forma parte, esto es, socializado, integrado en la institución y ejerciendo de elemento activo de esa agrupación humana. Para ello, desde la Universidad se debe favorecer la relación interpersonal con sesiones, por ejemplo de cineforum, deporte en equipo, voluntariado social, promoción del asociacionismo, charlas-coloquios sobre aspectos relacionados con la temática de la titulación, mesas redondas y debates sobre la realidad juvenil.

Esta labor podría recaer sobre estudiantes voluntarios, estudiantes del ámbito de los social (pedagogos, psicopedagogos, psicólogos, educadores sociales, trabajadores sociales, profesores de secundaria especialidad formación y orientación laboral, intervención sociocomunitaria, etc.) en su periodo de prácticas profesionales.

Los problemas administrativos y burocráticos de ámbito académico que se les presentan a los estudiantes inmigrantes también son detonantes del abandono estudiantil. Estos inconvenientes administrativos pueden abordarse desde varias posiciones. La primera de ellas consiste en habilitar un horario de atención administrativa, exclusivamente, para los trámites de estas poblaciones. La segunda posibilidad pasa por reunir, en jornadas concretas, a todos los estudiantes de origen inmigrante para realizar, en esos tiempos, la totalidad de sus trámites burocráticos administrativos. Una tercera opción pasa por designar estudiantes mentores que se ocupen de ayudar a los compañeros de origen inmigrante a solucionar las gestiones académicas. Como cuarta medida pueden organizarse jornadas iniciales de información y asesoría, contar con ayuda de personal voluntario, miembros de ONGs o compatriotas asentados ya en la Universidad.

Un elemento importante en el nuevo diseño intercultural de la Universidad española sería la elección de uno o varios delegados interculturales de curso. Esta nueva figura, uniendo sus esfuerzos a los del delegado tradicional del curso, velaría por la convivencia del grupo y serviría de nexo entre los estudiantes de distintas culturas. Debería ser un estudiante sensible, con capacidad de armonizar demandas y buscar puntos de encuentro dentro de un diálogo en igualdad de condiciones. Del mismo modo, sería conocedor de la burocracia administrativa universitaria, buen gestor, de carácter abierto y empático, socialmente sensible y comprometido. La función esencial de 
esta figura consistiría en promover la relación y convivencia entre los estudiantes para que fluyera con naturalidad, evitando que se produjesen reductos o guetos.

Conseguir aunar distintas nacionalidades en tareas conjuntas puede ser una perfecta clave de inclusión intercultural, para ello, el profesor, también ha de acomodar su función docente, entender la realidad intercultural que se vive en el aula y responder conforme a las demandas de la clase. Su talante tiene que ser de apertura y acogida, de enriquecimiento y reflexión, de contraste y síntesis de todas las realidades. La metodología que despliegue puede ser decisiva para lograr estos objetivos. A través del trabajo colaborativo y en grupo, en equipos, en parejas, en triángulos, la metodología universitaria debe crear un puzzle formativo, donde todas las piezas sean imprescindibles para alcanzar la finalización de la tarea previas. El estudiante inmigrante tiene que ser y sentirse una pieza más. Igual de necesaria e imprescindible para este fin que el autóctono.

En una universidad inclusiva, el nivel de exigencia, a la hora de calificar al universitario inmigrante, ha de ser idéntico al desplegado con el estudiante español. Si se hiciese alguna diferencia se estaría cayendo en una situación de discriminación positiva o negativa, pero siempre en una situación de singularidad manifiesta, que a la larga, resultaría dañina para la autoestima, autoimagen y aceptación del estudiante culturalmente diverso, e injusta para el estudiante autóctono. La inclusividad se diseña para el proceso formativo, no para su calificación.

Uniendo a estas medidas instructivas, organizativas, metodológicas y afectivas una buena política de becas basada en una actitud social compensatoria para todos aquellos estudiantes - tanto de origen inmigrante como autóctono- que no perciban rentas superiores a una cantidad prefijada se rebajaría el índice de abandono de los estudios universitarios. Estas acciones se enmarcarían en la filosofía de la justicia social distributiva y en el impulso a la cooperación internacional.

Es un derecho y un acto de justicia social nivelar desigualdades para optar al disfrute de los mismos derechos en igualdad de condiciones. La educación, es un derecho con una doble repercusión. Por un lado beneficia al individuo que la recibe y a su familia y, por otro lado, a la sociedad que ha formado al ciudadano educado y va a recibir de él la riqueza del capital humano que le ha ofrecido. Pero para materializar este elenco de buenos deseos se necesita algo bien pragmático: una buena política de becas.

Es obvio que las medidas de naturaleza económica para esta población son de suma importancia puesto que, una de las causas por las que aban- 
donan los estudiantes de origen inmigrante los estudios es por problemas económicos originados, muchas veces, por la pérdida del empleo, una situación laboral precaria, necesidad de enviar remesas a sus países de origen, carestía de vida, etc.

El Gobierno de España ha creado una gran bolsa de becas y subvenciones destinadas a estos estudiantes, entre ellas merece la pena reseñar las becas destinadas a iniciar estudios universitarios en España convocadas por la Secretaría de Estado de Educación y Formación (MEC, 2008b) con el objetivo de responder al principio de igualdad de oportunidades hacia los estudiantes que se encuentran en inferioridad de condiciones. La misma Secretaría de Estado ha convocado becas para los estudiantes que cursan estudios universitarios o superiores. Esta modalidad de beca cubre gastos de movilidad, residencia, material, desplazamiento o transporte.

Entre las subvenciones destinadas a estudiantes inmigrantes universitarios se encuentran las concedidas desde el Ministerio de Trabajo e Inmigración (2008b) a través de la Dirección General de Integración e Inmigración destinadas a Municipios, Mancomunidades de Municipios y Comarcas conducentes a programas innovadores que favorezcan la integración de inmigrantes.

Esta población también puede optar a la concesión de ayudas a las Asociaciones juveniles en el ámbito universitario nacional convocadas por la Secretaría de Estado de Universidades e Investigación del Ministerio de Educación (2007). Entre ellas merece especial atención todas las relacionadas o creadas por inmigrantes que se han multiplicado en los últimos años pasando de las 15 existentes en 2005 a las 90 actuales.

Por último, las Comunidades autónomas también ofrecen diversos planes de apoyo para la integración de la población estudiantil extranjera, así como algunas universidades como la UNED a través de su política de becas y ayudas al estudio para los estudiantes con menores recursos.

\section{CONCLUSIONES Y REFLEXIÓN FINAL}

La finalidad general de la investigación se ha centrado en responder a los objetivos formulados. Para ello se ha procedido al análisis cuantitativocualitativo y se han desarrollado nuevas competencias con la finalidad general de promover la inclusión social y laboral del colectivo de estudiantes inmigrantes universitarios. 
Es preciso señalar que todas las herramientas permiten al estudiante adquirir y expresar correctamente los conocimientos, destrezas, habilidades $\mathrm{y}$ valores, con independencia de su lugar de nacimiento.

Las conclusiones de este Estudio se centran en los resultados obtenidos de los análisis, así como en la propuesta de los apoyos que se ofrecen a los inmigrantes universitarios de origen inmigrante para tener éxito en sus estudios. Podemos señar las siguientes:

- Se ha identificado el perfil de la población de estudiantes inmigrantes universitarios: edad entre 20-30 años, predominando el sexo femenino; optan por estudios de larga duración y compatibilizan estudio y trabajo.

- Los estudiantes que obtiene mejores calificaciones suelen ser más jóvenes, mujeres y que dedican 15 horas semanales al estudio.

- Los motivos por los que se matriculan en una carrera universitaria en España han sido culturales y de formación, mayoritariamente.

- Como factores que inciden en el éxito de sus estudios cabe señalar los intereses, los recursos utilizados y las expectativas.

- Como recursos de apoyo, los inmigrantes universitarios señalan que precisan variedad de recursos personalizados y diferenciados para lograr el éxito.

- Como causas del abandono de los estudios universitarios, se mencionan las laborales y económicas; dificultad para conciliar estudio y trabajo.

El éxito académico que podría facilitarles una educación universitaria de tipo inclusivo redundaría en una integración social y laboral, más fácil, para estas poblaciones desfavorecidas.

Llama la atención de los investigadores que los estudiantes que han tomado parte en este estudio no hayan reseñado como necesidad, sin cubrir, el contar con textos escritos en sus idiomas maternos ni el reclamar mayores facilidades para homologar los estudios académicos obtenidos en sus países de origen. Estas peticiones se han hecho, reiteradas veces, en investigaciones sobre temáticas afines. Sin embargo, los resultados de esta investigación han sido bien concretos y tangibles a la vez que fácilmente abordables tanto desde las instituciones como desde las aulas universitarias. 
Estos resultados se convierten en un motivo que hace ver con verdadera esperanza la formación universitaria de estas poblaciones y la formación que puedan recibir, en este país, los estudiantes extranjeros que en los próximos años se desplacen por las universidades españolas, siguiendo las indicaciones del Plan Bolonia, sólo es cuestión de ponerse a hacerlo. 


\section{REFERENCIAS BIBLIOGRÁFICAS}

Arrimadas, I. y Hernando, A. (2007). Educar a los alumnos de todo el mundo, Rev. Escuela, 3.768.

Bugeda, J. (1974). Manual de técnicas de investigación social. Madrid: Instituto de Estudios Políticos.

Camarero (2007). Estudio de las demandas del colectivo de estudiantes inmigrantes matriculados en la UNED. MTAS-UNED.

Comisión Europea (2008a). Eurobarómetro 2008 Disponible en http://ec. europa.eu/public_opinion/index (consulta 2009; 10 de enero).

(2008b). Dirección General de Justicia, Libertad y Seguridad. La Haya Disponible en www.europa.eu/scadplus/leg/es y http://www.europol.europa.eu (consulta 2009, 20 de febrero).

Fernández, M. J. (2006). Necesidad de los estudiantes universitarios ante la realidad de la Educación Superior Europea. Madrid: MEC.

Fernández Enguita, M. y Terrén, E. (2008). De inmigrantes a minorías: temas y problemas de multiculturalidad. Revista de Educación..

Gil, M. (2008). Conferencia Regional para la educación Superior en América latina y El Caribe. Argentina: Universidad Nacional de Viña del Mar.

Guillén, M. F. y Ontiveros, E. (2007). La inmigración y las multinacionales españolas, El País 16/05/2007. Disponible en http://www.almendron.com/tribuna/index.php (consulta 2008, 16 agosto).

Jefatura del Estado. Ley Orgánica 6/2001, de 21 de diciembre, de Universidades. BOE núm. 307 de 24/12/2001. Dispo- nible en: http://www.boe./días/2001-1224/pdfs/A49400-49425.pdf (consulta 2009, 28 de diciembre).

Ministerio de Educación y Ciencia -MEC- (2008). Informe Datos Básicos del Sistema Universitario Español. Curso 2008/2009. Disponible en http:/www.micinn.es/univ/ccuniv/html/ estadistica (consulta 2009, 28 de diciembre).

(2009). Datos y Cifras del Sistema Educativo Español. Curso 2009-2010. Disponible en http://www.educacion.es (dctm/ministerio/educacion (consulta 2010, 23 de mayo).

(2007). Plan Estratégico de Ciudadanía e Integración 2007-2010. Secretaría de Estado de Inmigración y Emigración.

Ministerio de Educación, Política Social y Deporte (2008). Resolución de 2 de junio de 2008 de la Secretaría de Estado de Educación y Formación.

Ministerio de Trabajo e Inmigración (2008a). Observatorio español. Disponible en http://oberaxe.es. (consulta 2009, 24 de noviembre).

(2008b). Resolución de 11 de agosto de 2008 de la Dirección General de Integración de los Inmigrantes (BOE núm. 211 de 1 de septiembre, p. 35761).

Oliver, J. y otros (2004). La inmigración parará la caída del alumnado universitario. Aula intercultural. Disponible en http://www.aulaintercultural.org (consulta 2009, 12 de diciembre).

Pérez, M. G. y otros (2008). Hacia una universidad Inclusiva: medidas de apoyo al alumnado inmigrante. Programa de Estudios y Análisis 2008. MICINN. 
Pérez, M. y Desrues, T. (2007): Opinión de los españoles en materia de racismo y xenofobia. MTAS- Dirección General de Integración de los inmigrantes. Observatorio Español del Racismo y la Xenofobia. IESA/CSIC.

Proyecto Europeo de Redes Comenius INCLUES. Llaves para una educación
Inclusiva y Cognitiva, Disponible en www.inclues.org. (consulta 2009, 14 de octubre).

Tagliacarne. Tabla-prontuario. Disponible en http://www.tesisen xarxa.net (consulta 2009, 28 de diciembre). 


\section{PALABRAS CLAVE} tivos.

Inmigración, Universidad inclusiva, Éxito académico, Apoyos educa-

\section{KEY WORDS}

Immigration, Inclusive University, Academic success, Educational support.

\section{PERFIL ACADÉMICO Y PROFESIONAL DE LAS AUTORAS}

$\mathrm{M}^{\mathrm{a}}$ Paz Lebrero Baena, Doctora en Pedagogía por la Universidad Complutense de Madrid, Profesora Titular en la UNED desde 1986. Miembro de los Grupos de Investigación "Intervención socioeducativa" (G44Edu16) y "Centro de Investigación MANES" (G40Edu15). Sus publicaciones y actividad investigadora se centran en diferentes ámbitos de pedagogía social (menores en riesgo, atención socioeducativa, etc.) y de educación infantil, por cuyos materiales ha recibido premios nacionales e internacionales.

$\mathrm{M}^{\mathrm{a}}$ Pilar Quicios García, Doctora en Filosofía y Ciencias de la Educación por la UNED, Profesora en la Facultad de Educación de dicha la Universidad. Miembro de diferentes Grupos de Investigación, autora e investigadora principal de publicaciones y Proyectos de Investigación sobre riesgo social financiados por AECI y por FIDE, así como el Proyecto de Investigación Internacional auspiciado por el Ministerio de Educación chileno "La calidad docente factor de prevención del Riesgo Social”.

Dirección de las autoras: $\mathrm{M}^{\mathrm{a}}$ Paz Lebrero Baena y $\mathrm{M}^{\mathrm{a}}$ Pilar Quicios García UNED. Facultad de Educación. Dpto. Teoría de la Educación y Pedagogía Social.

Paseo Senda de del Rey, n. ${ }^{\circ} 7$ (28040) Madrid

E-mail: mplebrero@edu.uned.es pquicios@edu.uned.es

Fecha Recepción del artículo: 5. Abril. 2010

Fecha Revisión del artículo: 27. Mayo. 2010

Fecha Aceptación del artículo: 6. Julio. 2010 